\title{
Bioinformatics of the sugarcane EST project
}

\author{
Guilherme P. Telles*, Marília D.V. Braga, Zanoni Dias, Tzy-Li Lin, José A.A. Quitzau, \\ Felipe R. da Silva and João Meidanis
}

Abstract

The Sugarcane EST project (SUCEST) produced 291,904 expressed sequence tags (ESTs) in a consortium that involved 74 sequencing and data mining laboratories. We created a web site for this project that served as a 'meeting point' for receiving, processing, analyzing, and providing services to help explore the sequence data. In this paper we describe the information pathway that we implemented to support this project and a brief explanation of the clustering procedure, which resulted in 43,141 clusters.

\section{INTRODUCTION}

The application of expressed sequence tag (EST) technology has proven to be an effective tool for gene discovery (Adams et al., 1991), gene mapping (Schuler, 1997) and the generation of gene expression profiles (Boguski and Schuler, 1995).

EST projects are usually conducted by a single laboratory, which prepares the cDNA libraries, isolates and sequences clones, analyzes the data and submits it to GenBank. However, the Sugarcane EST project (SUCEST) involved the cooperation of 24 sequencing laboratories, a bioinformatics laboratory, a coordinating laboratory, 50 data mining groups scattered throughout Brazil and an international relations group. A new Brazilian bioinformatics group also became associated with the project during a later phase. Starting early in 1999, in 15 months the SUCEST project generated 291,904 sequences from 260,352 clones from 37 different libraries.

Brazilian genome research has been consortium-based since its first project, the sequencing of the complete genome of the phytopathogenic bacterium Xylella fastidiosa (Simpson et al., 2000), conducted by the Organization for Nucleotide Sequencing and Analysis (ONSA network). Although a consortium-based genome project provides a larger number of researchers, technicians and sequencing machines it demands a much more organized data flow. In the SUCEST project, the Bioinformatics Laboratory (Laboratório de Bioinformática - LBI) was responsible for receiving data from a network of sequencing laboratories, assessing quality, storing and clustering the data, and providing many other services. In this paper these tasks are described in some detail and quantitative figures from the project are given.

\section{METHODS}

Computational systems

For a short time in the beginning of the project, the SUCEST web site was hosted by a personal computer with 128 MB of memory running the Linux operating system (Red Hat 6.2) but now the site resides on a Compaq AlphaServer ES40 with two Alpha $667 \mathrm{MHz}$ processors, 8 GB of RAM and 384 GB of hard-disk storage space running OSF-1 operating system version 4.0G. However, the bulk of the project was executed on a Compaq AlphaServer DS20 with two Alpha $500 \mathrm{MHz}$ processors, $4 \mathrm{~GB}$ of RAM and 144 GB of hard-disk storage space running OSF-1 version $4.0 \mathrm{~F}$. Since this was the system on which most of the tools were developed we will concentrate on it for the rest of the paper.

The Web engine server is Apache (www.apache.org) version 1.3.9. Programs were written in Perl version 5.005 (www.cpan.org), and PHP version 3.0.12 (www.php.net). The database management system is MySQL version 3.22.26a (www.mysql.com).

Input data consisted of data received through web forms, including chromatograms produced by ABI 377 sequencing machines (Applied Biosystems), and data mining reports in HTML format.

The base calling and sequence extraction programs used were phred version 0.980904.e (www.phrap.org) and phd2fasta version 0.990622.d (www.phrap.org). The sequence comparison programs used were cross-match version 0.990319 (www.phrap.org) and blastall version 09/19/1999 (www.ncbi.nlm.nih.gov) that implements the BLAST algorithm (Altschul et al., 1997). Assembly programs were phrap version 0.990319 (www.phrap.org) and CAP3 (Huang and Madan, 1999). Off-the-shelf scripts 
were used to provide search by keywords in the reports produced by data mining groups, database administration and other minor tasks. Each piece of software used is either free for academic purposes or was developed by our team.

\section{Computational methods}

From a computational point of view, SUCEST may be seen as a large data repository and as a provider of Internet-based services for a community of different users. Figure 1 shows the major relationships between users, services, data and programs in the project.

There are several types of users: members of sequencing laboratories who submit chromatograms from clone libraries, members of data mining laboratories who perform searches on the project database and publicize their results in data mining reports, and members of the project coordination team who monitor the status of the project and the distribution and validation of control plates. These users interact with data through services that add to, retrieve from, and update the data repositories.

Data include sugarcane ESTs, information about project members, data mining reports, control data, summaries and the output from programs that perform automated searches in databases, organize the sequences into clusters and the clusters into categories. In the following paragraphs we describe the users, data, and SUCEST services and programs, showing how they interact.

\section{DEFINITIONS}

\section{Objects}

In the SUCEST project data is stored in two different kinds of repositories: operating system directories and a relational database. The directories hold biological sequence files, results from BLAST and cross-match searches in biological databases, and data mining reports. Biological se-

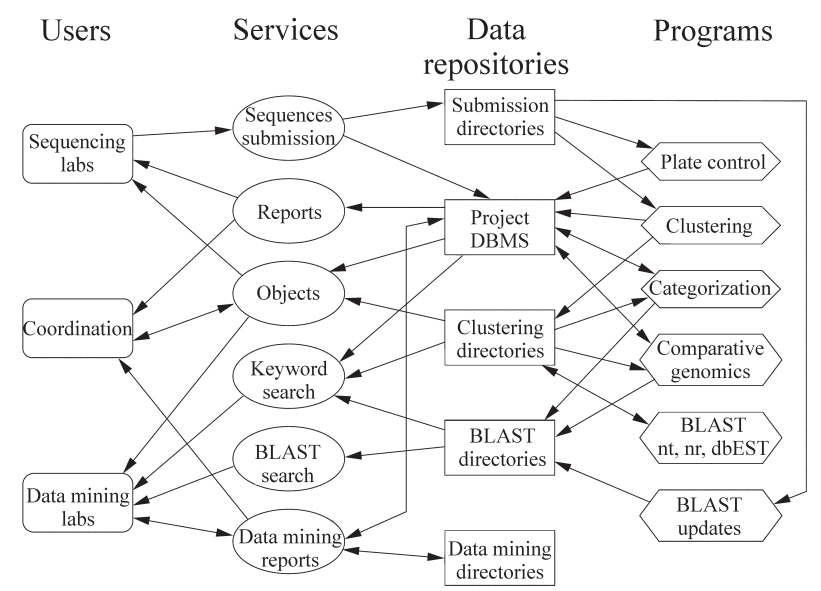

Figure 1 - Major relationships between users, services, data and programs involved in the SUCEST project. Arrows indicate the flow of information. quence files include chromatograms, files in a standard format called fasta format (www.ncbi.nlm.nih.gov/ BLAST/fasta.html), quality files, and files generated by clustering, categorization and comparative genomics procedures. The project uses only one relational database, with several interconnected tables that store other biological and management data, e.g. libraries, sequencing plates and data on laboratories and their members. The database also points to data in directories. The major entities (objects) in our database are described below, where we also introduce quantitative figures and details from the project's pipeline.

\section{Laboratories}

There are 78 laboratories involved in the SUCEST project that belong to one or more of five groups: the DNA Coordination Group, the Bioinformatics Group, the Data Mining Group, the Sequencing Group and the International Cooperation Group. Each participating laboratory is identified by a two-letter code. The services and data that a member of a particular laboratory can access depend on the group to which the laboratory belongs. A member of each laboratory is designated as being the head of the unit involved in SUCEST-related work and receives notification of some of the activities performed by the laboratory members.

\section{Members}

A SUCEST member is a person who belongs to at least one laboratory. Several members belong to both a sequencing laboratory and a data mining laboratory. Data held on members include their name, the laboratories to which they belong, their e-mail address, phone numbers and a login name and password to grant access to authorized services. SUCEST had 256 members as at March 25, 2001.

\section{Libraries}

The ESTs included in the SUCEST database came from 37 different libraries prepared from different sugarcane tissues under different conditions (Vettore et al., 2001). The name and description of the library and vector employed in cloning were recorded for each library. Each library received a two-letter code indicating the tissue from which the library was derived, together with a consecutive number assigned for every new library derived from the same tissue. For example, LR1 indicates that the library came from leaf roll (LR) with long inserts (library 1) while LR2 shows that the library came from leaf roll (LR) with small inserts (library 2). There are three possibilities for the status of each library: 'test' for validating libraries, 'start' for libraries released for sequencing and 'stop' when the DNA Coordination Group decides it is not worth continuing to sequencing a distributed library. Of the 37 libraries prepared for the project, 32 were started and 5 were aban- 
doned after the 'test' phase. Those not formally started either produced too much redundancy or very small reads.

\section{Plates}

SUCEST clones are organized in 96-well plates that hold clones from the same library in an $8 \times 12$ grid. Sequencing is done for a whole plate and the data is sent to the LBI for processing and storage. Data for a plate include the library that it came from and the laboratory that is authorized to send data on this plate. A plate has a three-digit identification tag, except for control plates (see below), which have the letter ' $C$ ' and two digits. The SUCEST database holds data from 2,771 different plates.

\section{Reads}

Reads are the same as ESTs and are extracted using the phred program from chromatograms submitted by the sequencing laboratories and screened for vectors with the cross-match program. All reads are stored in directories as chromatogram files and also as a pair of text files holding the sequence and its quality in fasta format. For every read the following attributes are stored in the database: the plate and the position on the plate where the read came from; information about the submission process (e.g. date and time of submission); the number of vector and non-vector bases with phred quality equal to or higher than 20; the number of vector and non-vector bases with phred quality less than 20; the starting and ending positions for every vector sequence identified in the read and whether or not the read has relevant data (see preparation sheet below.)

Every read has a name that is a concatenation of its laboratory, library and plate codes, plate position and read direction ( $5^{\prime}$ or 3 '). For example, reading from right to left, the string SCACAD1001A01.g is the name for the 5' read (3' uses .b as a suffix.) of the clone in well A01 of plate 001 of library AD1, sequenced by laboratory AC. The prefix SC stands for sugarcane. Every position on the plate is identified by its row (A to $\mathrm{H}$ ) and column (01 to 12$)$.

\section{Preparation sheet}

Before a laboratory can sequence and submit a plate, it must provide a sheet of information about the process used to prepare the plate. There are records in the database for every well where bacteria did not grow and for the wells from which it was not possible to obtain DNA. Every well marked as a problem corresponds to a sequence without information relevant to the project.

\section{Control plates}

For every set of 12 plates a control plate is built using the $8^{\text {th }}$ column of each controlled plate, so 12 columns make one control plate that is sequenced. The sequences from both control and controlled plates are compared against each other using cross-match, and the matches are stored in the database. A criterion, based on the matches distribution over the control and controlled plates, was established to automatically mark plates that probably had tracking and naming errors due to plate preparation and sequencing processes. Matches distributions could be visualized via a web service, and plates with problems could be fixed and resubmitted by the laboratory that produced them.

\section{Clusters}

SUCEST reads are grouped by the clustering procedure described below, which creates sets of aligned reads that we call clusters. In our database we store the reads that are part of each cluster. Moreover, in addition to being a set of reads, a cluster has an alignment and a consensus sequence. Alignments, consensus sequences, and quality files are stored in cluster directories. A cluster also has a name, which is equal to the name of oldest read in the cluster.

\section{Services and programs}

Data enter and are retrieved from the SUCEST data repository through a set of services available on web pages hosted at LBI. Data is also generated within the LBI by programs that are executed either automatically or manually. Brief descriptions of these services and programs are presented below and provide a general overview on how the SUCEST web site is organized and how it works.

\section{Data retrieval}

Data is retrieved from the SUCEST database in units called 'objects' which are the same as the data entities described above under 'Definitions'. Each object has its own web page containing information about the object and links to any other object, service or report directly related to it. Starting from a laboratory or library object it is possible to reach the web page of any other object. Some objects point to pages that include data extracted from the directory structure of the project. For instance, one can visualize reads and its qualities in many versions: immediately after submission but before screening, after screening but before trimming (see below under 'Clustering and Trimming') and after trimming. For clusters, it is possible to see the reads in a cluster and their alignments, including the consensus.

An object search service was created to allow direct access to any object. Given the code and the type of the object, the service delivers its page. For the 'Member' object type it is possible to search by name, email, department, city or institution.

Besides objects, some reports that summarize data are also available for the project: the Summary of Submitted Reads gives totals per laboratory or per library of submitted, payable and clusterizable reads, and the Summary of Control Plates gives the totals of accepted and rejected control plates. 
SUCEST database users who are SQL (Structured Query Language) literate may take advantage of a service that allows generic queries to the database. Queries can be typed in a web form and the results are returned in tabular fashion. Entity-relationship diagrams and table descriptions for our database are available to help users in this task.

\section{Sequences submission}

Sequences are submitted by sequencing laboratories only, the submission process requiring the user to access the project's web site using a valid login/password pair to upload a set of 96 chromatograms (i.e. one plate). When an upload finishes certain pre-requisites are verified: all chromatograms must belong to the same plate, the laboratory that is trying to submit a plate must be the one authorized to do so, the preparation sheet for that plate must have already been submitted and the reads must be in accordance with the naming conventions.

If the pre-requisites are satisfied, the phred and phd2fasta programs are used to extract the sequences and their qualities in fasta format from chromatograms and the cross-match program is used to mask vector sequences in the reads. These steps take only a few minutes (this time has essentially been constant during the project because the analysis done upon submission does not depend on the other reads present in the repositories).

After submission analysis, a report that summarizes the process and the sequences received is presented to the submitter who is asked to confirm the submission or not. If the submission is confirmed, the database is updated and if there is an older version of the plate it is replaced. Directories are updated as well. If the submission is not confirmed (e.g., if the submitter is not happy with the quality assessment) the submission is discarded.

Figure 2 shows the path followed by a read in the LBI, starting from the submission. The submission procedure corresponds to the part of the figure starting at 'Zip file', extending through top line and reaching the 'Report Generator'. Other steps in the diagram are performed by programs described in the following sections.

\section{Clustering and trimming}

Clustering of ESTs is important to reduce the amount of sequence data that miners have to look at, and to orga-

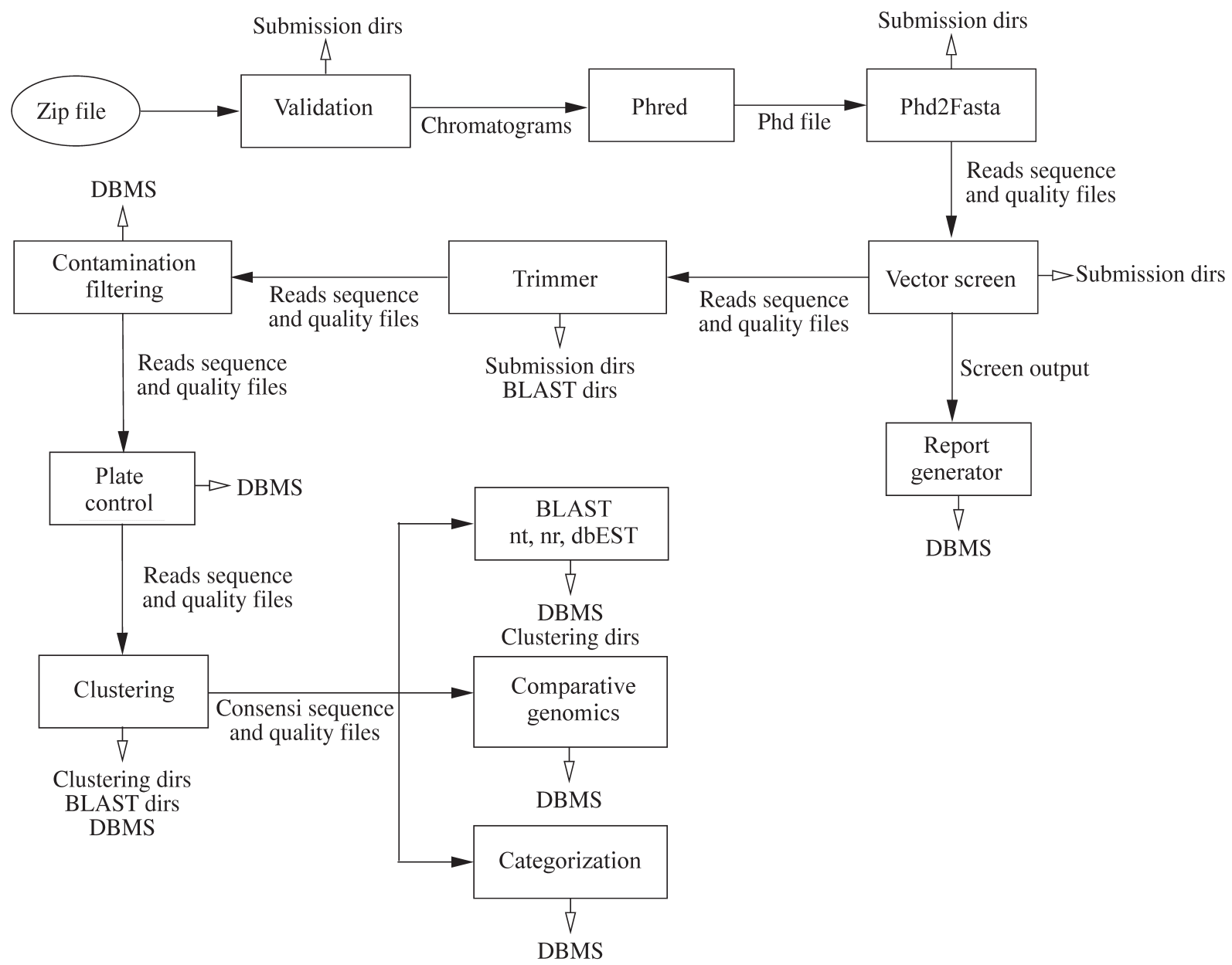

Figure 2 - The operations taking place on a read in the SUCEST pipeline. Black arrows linking boxes indicate data that flow from one stage to the next while white-headed arrows going out of boxes indicate date repository updates. 
nize the reads in a less redundant set. In the SUCEST database, clustering had as an additional motive the need to estimate the level of redundancy in the libraries.

Early on two pivotal decisions were made, the first being that each cluster should reflect a transcript rather than a gene, allele or other biological entity while the second was that a cluster consists not only of a set of reads but also of an alignment of these reads.

In this context, our first scheme was to group similar transcripts and to produce consensus sequences using the assembly program phrap. This strategy was sufficient in the early stages of the project but, as data accumulated, a series of problems forced us to change the scheme, as described below.

To minimize artifacts, reads were trimmed before clustering. This trimming procedure started with vector masking using the cross-match program followed by removal of some of the poly-A, vector and adapter regions. A quality trimmer was also applied, removing bases from the ends of the sequence one by one until there were at least 12 bases with phred quality above 15 in a window of 20 bases at the end. Reads were also checked for contamination against Xylella fastidiosa, Xanthomonas citri, Escherichia coli and other potential contaminants that could be present in the laboratories that produced the libraries. BLAST was used to compare the reads and potential contaminators and if a match of at least 100 bases and more than $90 \%$ identity occurred the read was marked as probably being due to contamination. However, marked reads were kept in clustering and subsequent analyses to allow data miners to decide for themselves whether or not a specific read was contaminated.

Trimmed reads were assembled using the phrap program with quality and stringent arguments (-penalty -15 -bandwidth 14 -minscore 100 -shatter_greedy). Every contig and singlet produced by phrap was taken as a cluster. As new plates came in, a program automatically updated the database, directories and BLAST results for every cluster that changed and was already in the database. Initially, clustering was performed every day but as the set of sequences grew the updates became sparser, running once a week. In the final phases of the project, clustering would typically occupy an entire processor for about 20 hours.

The last assembly done with phrap included 261,609 trimmed reads and produced 81,223 clusters. However, changes were made due to remarks made by several members of the project that the total number of clusters in the database was unreasonably large, that many clusters were malformed and that some clusters appeared as if they could be combined. These changes are described in detail by Telles and da Silva (2001). The new scheme was based on careful testing and evaluation, and consisted of a more elaborate trimming procedure, the use of the CAP3 assembler (Huang and Madan, 1999), which is the same tool used to produce TIGR's gene indices (Quackenbush et al., 2000). Trimming in this new procedure included ribosomal RNA removal, comprehensive removal of poly-A, poly- $\mathrm{T}$, vector and adapter regions and improved low-quality-end trimming. CAP3 was fed with 237,954 reads and their quality data and produced 43,141 clusters.

Both clustering versions are accessible through the project web site, with data from both methods available for most services.

\section{Keyword search}

Keyword search is a service that allows users to search for a set of keywords in the header lines of every sequence in NCBI's nr, nt and dbEST databases (www.ncbi. nlm.nih.gov) that hits any cluster in SUCEST. To perform a query the user gives a database name (nr, nt or dbEST), a logical expression of keywords (that may include 'or' and 'and' connectors) and the maximum e-value required (an optional parameter which defaults to $1 \mathrm{e}-5=10^{-5}$ ). The service then returns the clusters that have a hit with the expected or better e-value, and whose subject heading contains words satisfying the logical expression. The resulting list of clusters is ordered by e-value.

A program was created for keeping BLAST results against nr, nt and dbEST up to date for all SUCEST clusters. A BLAST result against a certain database is considered outdated for a SUCEST cluster if the cluster was newer than the result or if the cluster or the database were modified after the last BLAST run. When the program finds outdated BLAST results it builds a queue giving priority to older clusters. If the databases are on different computers the system is able to reduce the processing time by running several BLASTs in parallel (one on each remote server) and takes about 2 or 3 days. If the databases are on a single computer, BLAST searches take considerably longer.

\section{Subclustering}

This service is used to evaluate statistics about subsets of clusters obtained by clustering, including read frequency by cluster size, total reads, total clusters, redundancy and novelty. To select the subset of clusters, the user has to indicate the reads that belong to the clusters. Any cluster that contains a read in the selection is included in the evaluation. To locate reads, one or more elements (laboratory, library, plate, position and direction) from their names should be selected, e. g. selecting a particular laboratory will generate the statistics for the clusters that have at least one read sequenced by that laboratory.

\section{BLAST search}

A BLAST service allows searches against SUCEST reads, reads in their trimmed version and cluster consensi. These databases were updated automatically on a daily basis to incorporate new reads and consensi. 
Data mining report

Data mining groups submit HTML formatted reports to the SUCEST site and update them periodically. Users may access reports through an index page that provides access to the reports of every data mining group and a keyword search is also available. When a report archive is uploaded a service takes care of unpacking the files and updating the index page and the search index. Information about reports is also kept in the SUCEST database, including the name and a summary of the project, its members and a submission date and submitter name.

\section{Categorization}

SUCEST members tried to categorize the clusters in the project, in an attempt to determine their function and to aggregate information. Thirty categories were defined, and 32,438 proteins with known function were selected from public databases to serve as examples in each category. Public databases included MIPS Arabidopsis thaliana database (mips.gsf.de), Clusters of Orthologous Groups - functional annotation (www.ncbi.nlm.nih.gov/COG/), EGAD cellular roles (www.tigr.org/docs/tigr-scripts/egad_scripts /role_report.spl) and others.

Categorization was achieved by two methods: automatic and manual. In automatic categorization a database was constructed containing the proteins selected from public databases and a BLAST search was performed against this database using SUCEST clusters as input. Any cluster was considered to be in category $\mathrm{X}$ if it matched a category $\mathrm{X}$ sample protein with an e-value better than or equal to $10^{-10}$ and covered $70 \%$ or more of the example. A cluster could be in many different categories. This method categorized $36 \%$ of the 43,141 clusters. For manual categorization a web service was built to allow manual annotation when automatic annotation produced ambiguous categorization or produced no categorization at all. Based on BLAST results against the nr database, SUCEST members were able to establish a direct relation between a cluster and a category. Manual annotation significantly increased the number of categorized clusters and as of March $20^{\text {th }}, 2001$, $60.5 \%$ of the clusters were categorized.

\section{Comparative genomics}

To obtain information on sugarcane and its relationship to other species, SUCEST cluster consensi were compared against other organisms. The first organism selected for comparison was the model plant Arabidopsis thaliana. Every cluster consensus was BLASTed against $A$. thaliana chromosomes, proteins and ESTs. Clusters that produced no matches against $A$. thaliana, were also BLASTed against ESTs from Lycopersicon esculentum, Glycine max, Lotus japonicus, Hordeum vulgare, Oryza sativa, Sorghum bicolor, Zea mays, Triticum aestivum and Medicago truncatula. Results from these searches were inserted in our database, allowing queries to determine the distribution of these hits per library, per cluster, or some other grouping criteria.

\section{Management}

These services provide a way for the DNA Coordination Group to input management information into the SUCEST database. This information is used mainly by services that perform checking and summarizing operations. Using the library management services, the DNA Coordination Group modifies the status of any library and assigns plates to sequencing laboratories. Manual plate approval is also possible via a service that displays control and controlled plates showing which cells match in control and controlled plates.

\section{DISCUSSION}

A key aspect of the project was the close interaction between the biological laboratories and the LBI. Discussion lists or telephone calls were used so that users could give suggestions for new services and quickly point out problems with the services (broken links, bugs, etc.) This daily, intensive interaction was undoubtedly one of the main reasons for the success of the project.

Clustering started early and had a dramatic impact during the project. Re-clustering on a regular basis demanded designing and implementing programs to update databases and BLAST results against the nr, nt and dbEST databases, and also used a lot of processor time. When another clustering scheme was adopted the web site had to change to accommodate both versions simultaneously and to show relationships between clusters in different versions and both bioinformatics and data mining staff needed some time to adapt to the changes.

The two most important lessons learnt during the SUCEST project were 'avoid changing systems' and 'keep reference sequences, not cluster lists' which we will discuss in more detail in the following paragraphs.

Avoiding changes in the systems is important. During this project we had to change the underlying computing system twice, the first time from a personal computer to a medium-sized server and then from this to a larger server. These changes caused many problems, e.g. programs that used to work on one system would not work on the other system, users had to get used to new addresses etc. The migration process proved time-consuming and error-prone. Our advice would be to set up a system that is big enough right from the start and keep the project there for as long as possible. To minimize the impact of migration it is important to devise the directory structure in a system-independent way, for instance data can be placed in directories that will not conflict with system directories and programs can be installed in standard locations and execution path variables used to assure they will work. Another important piece of advice is to use software that combines many phys- 
ical disks into one big volume of, say, a few hundred gigabytes. Most vendors provide such software for a small fee.

It is also important to keep reference sequences instead of lists of clusters. In this project, data accumulated at a fast rate and clustering was redone frequently. Some data mining groups had problems trying to keep up with the frequent updates because they maintained lists of relevant clusters. Each time the clustering was redone some clusters would disappear (merge into larger ones) or the read composition of a cluster would change, requiring a lot of manual labor. Our advice would be to use reference sequences from Genbank or another stable sequence database, which can then be used as queries to retrieve the cluster lists via BLAST. Proceeding in this way lists can be quickly reconstructed from the reference sequences using automated methods.

There are many other programs, not presented here, that contribute to the functionality of the SUCEST web site. Some services and programs have already been disabled (e.g. the sequence submission and plate control programs) but others, such as the keyword search, BLAST and report submission programs are still being used by data mining laboratories and will be used by the international community when the web site goes public. This will certainly transform the meeting point of the project's community into the meeting point of a wider group which will produce new demands for services and data storage.

\section{ACKNOWLEDGMENTS}

This work was supported by FAPESP, CNPq and COPERSUCAR.

\section{RESUMO}

O projeto SUCEST (Sugarcane EST Project) produziu 291.904 ESTs de cana-de-açúcar. Nesse projeto, o Laboratório de Bioinformática criou o web site que foi o "ponto de encontro" dos 74 laboratórios de sequenciamento e data mining que fizeram parte do consórcio para o projeto. O Laboratório de Bioinformática (LBI) recebeu, processou, analisou e disponibilizou ferramentas para a exploração dos dados. Neste artigo os dados, serviços e programas implementados pelo LBI para o projeto são descritos, incluindo o procedimento de clustering que gerou 43.141 clusters.

\section{REFERENCES}

Adams, M.D., Kelley, J.M., Gocayne, J.D., Dubnick, M., Polymeropoulos, M.H., Xiao, H., Merril, C.R., Wu, A., Olde, B., Moreno, R., Kerlavage, A.R., McCombie, W.R., and Venter, J.C. (1991). Complementary DNA sequencing: "expressed sequence tags" and the human genome project. Science 252: 1651-1656.

Altschul, S.F., Madden, T.L., Schäffer, A.A., Zhang, J., Zhang, Z., Miller, W. and Lipman, D.J. (1997). Gapped BLAST and PSI-BLAST: a new generation of protein database search programs. Nucleic Acids Res. 25: 3389-3402.

Boguski, M. and Schuler, G. (1995). ESTablishing a human transcript map. Nature Genetics. 10: 369-371.

Huang, X. and Madan, A. (1999). CAP3: A DNA sequence assembly program. Genome Res. 9: 868-877.

Quackenbush, J., Liang, F., Holt, I., Pertea, G., and Upton, J. (2000). The TIGR Gene Indices: reconstruction and representation of expressed gene sequences. Nucleic Acids Res. 28 (1): 141-145.

Schuler, G. (1997). Pieces of the puzzle: Expressed sequence tags and the catalog of human genes. $J$ Mol Med. 75 (10): 694-698.

Simpson, A.J.G., Reinach, F.C., Arruda, P., Abreu, F.A., Acencio, M., Alvarenga, R., Alves, L.M.C., Araya, J.E., Baia, G.S., Baptista, C.S., Barros, M.H., Bonaccorsi, E.D., Bordin, S., Bove, J.M., Briones, M.R.S., Bueno, M.R.P., Camargo, A.A., Camargo, L.E.A., Carraro, D.M., Carrer, H., Colauto, N.B., Colombo, C., Costa, F.F., Costa, M.C.R., Costa-Neto, C.M., Coutinho, L.L., Cristofani, M., Dias-Neto, E., Docena, C., El-Dorry, H., Facincani, A.P., Ferreira, A.J.S., Ferreira, V.C.A., Ferro, J.A., Fraga, J.S., Franca, S.C., Franco, M.C., Frohme, M., Furlan, L.R., Garnier, M., Goldman, G.H., Goldman, M.H.S., Gomes, S.L., Gruber, A., Ho, P.L., Hoheisel, J.D., Junqueira, M.L., Kemper, E.L., Kitajima, J.P., Krieger, J.E., Kuramae, E.E., Laigret, F., Lambais, M.R., Leite, L.C.C., Lemos, E.G.M., Lemos, M.V.F., Lopes, S.A., Lopes, C.R., Machado, J.A., Machado, M.A., Madeira, A.M.B.N., Madeira, H.M.F., Marino, C.L., Marques, M.V., Martins, E.A.L., Martins, E.M.F., Matsukuma, A.Y., Menck, C.F.M., Miracca, E.C., Miyaki, C.Y., Monteiro-Vitorello, C.B., Moon, D.H., Nagai, M.A., Nascimento, A.L.T.O., Netto, L.E.S., Nhani Jr., A., Nobrega, F.G., Nunes, L.R., Oliveira, M.A., de Oliveira, M.C., de Oliveira, R.C., Palmieri, D.A., Paris, A., Peixoto, B.R., Pereira, G.A.G., Pereira Jr., H.A., Pesquero, J.B., Quaggio, R.B., Roberto, P.G., Rodrigues, V., de M. Rosa, A.J., de Rosa Jr., V.E., de Sa, R.G., Santelli, R.V., Sawasaki, H.E., da Silva, A.C.R., da Silva, F.R., da Silva, A.M., Silva Jr., W.A., da Silveira, J.F., Silvestri, M.L.Z., Siqueira, W.J., de Souza, A.A., de Souza, A.P., Terenzi, M.F., Truffi, D., Tsai, S.M., Tsuhako, M.H., Vallada, H., Van Sluys, M.A., Verjovski-Almeida, S., Vettore, A.L., Zago, M.A., Zatz, M., Meidanis, J. and Setubal, J.C. (2000). The genome sequence of the plant pathogen Xylella fastidiosa. Nature 406: 151-157.

Telles, G.P. and da Silva, F.R. (2001). Trimming and clustering sugarcane ESTs. Genetics and Molecular Biology 24 (1-4): 17-23.

Vettore, A., da Silva, F.R., Kemper, E. and Arruda, P. (2001). The libraries that made SUCEST. Genetics and Molecular Biology 24 (1-4): 1-7. 